\title{
Use of a novel ball-joint guide array for magnetic resonance imaging-guided cannula placement and convective delivery: technical note
}

\author{
Krystof S. Bankiewicz, MD, PhD, ${ }^{1}$ Tomasz Pasterski, MD, ${ }^{2}$ Daniel Kreatsoulas, MD,1 \\ Jakub Onikijuk, MD, ${ }^{2}$ Krzysztof Mozgiel, MD, ${ }^{2}$ Vikas Munjal, BS, ${ }^{1}$ J. Bradley Elder, MD, \\ Russell R. Lonser, MD, ${ }^{1}$ and Mirosław Zabek, MD, PhD²
}

${ }^{1}$ Department of Neurological Surgery, The Ohio State University Wexner Medical Center, Columbus, Ohio; and ${ }^{2}$ Department of Neurological Surgery, Centrum Medyczne Kształcenia Podyplomowego, Brodno Hospital, Warsaw, Poland

OBJECTIVE The objective of this study was to assess the feasibility, accuracy, effectiveness, and safety of an MRIcompatible frameless stereotactic ball-joint guide array (BJGA) as a platform for cannula placement and convectionenhanced delivery (CED).

METHODS The authors analyzed the clinical and imaging data from consecutive patients with aromatic L-amino acid decarboxylase $(A A D C)$ deficiency who underwent infusion of adeno-associated virus (AAV) containing the $A A D C$ gene (AAV2-AADC).

RESULTS Eleven patients (7 females, 4 males) underwent bilateral MRI-guided BJGA cannula placement and CED of AAV2-AADC (22 brainstem infusions). The mean age at infusion was $10.5 \pm 5.2$ years (range 4-19 years). MRI allowed for accurate real-time planning, confirmed precise cannula placement after single-pass placement, and permitted on-the-fly adjustment. Overall, the mean bilateral depth to the target was $137.0 \pm 5.2 \mathrm{~mm}$ (range 124.0-145.5 mm). The mean bilateral depth error was $0.9 \pm 0.7 \mathrm{~mm}$ (range $0-2.2 \mathrm{~mm}$ ), and the bilateral radial error was $0.9 \pm 0.6 \mathrm{~mm}$ (range 0.1-2.3 mm). The bilateral absolute tip error was $1.4 \pm 0.8 \mathrm{~mm}$ (range $0.4-3.0 \mathrm{~mm}$ ). Target depth and absolute tip error were not correlated (Pearson product-moment correlation coefficient, $r=0.01$ ).

CONCLUSIONS Use of the BJGA is feasible, accurate, effective, and safe for cannula placement, infusion MRI monitoring, and cannula adjustment during CED. The low-profile universal applicability of the BJGA streamlines and facilitates MRI-guided CED.

https://thejns.org/doi/abs/10.3171/2020.6.JNS201564

KEYWORDS ball-joint guide array; convection-enhanced delivery; drug delivery; gene therapy; magnetic resonance imaging; stereotactic; surgical technique

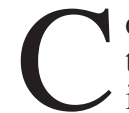

ONVECTION-ENHANCED delivery (CED) of putative therapeutics to perfuse targeted brain regions has increased significantly. Specifically, frameless stereotactic cannula placement and real-time MRI of perfusion are used to treat diseased brain circuits/anatomy in an expanding variety of disorders, including Parkinson's disease, Huntington's disease, Alzheimer's disease, metabolic disorders, aromatic L-amino acid decarboxylase (AADC) deficiency, and neurooncology. ${ }^{1-10}$ Currently, there are two frameless stereotactic systems that are compatible with an MRI scanner (Nexframe, Medtronic; and ClearPoint, ClearPoint Neuro Inc.) and have been adapted for cannula placement, infusion imaging, and cannula adjustment during CED. ${ }^{4,11-13}$ However, these systems require dedicated software and have large profiles that can prohibit contemporaneous device placement, simultaneous infusions, and/or effective targeting of multiple brain structures.

Recently, nonhuman primate data ${ }^{14}$ have indicated that a frameless stereotactic ball-joint guide array (BJGA) may provide a versatile, smaller-profile, lower-cost platform that supports a more streamlined approach for MRI-guided infusion cannula placement, infusion monitoring, and cannula adjustment during CED. To assess the clinical

ABBREVIATIONS AADC = aromatic L-amino acid decarboxylase; $\mathrm{AAV}=$ adeno-associated virus; $\mathrm{BJGA}=$ ball-joint guide array; $\mathrm{CED}=$ convection-enhanced delivery; PEEK = polyetheretherketone.

SUBMITTED April 30, 2020. ACCEPTED June 16, 2020.

INCLUDE WHEN CITING Published online October 23, 2020; DOI: 10.3171/2020.6.JNS201564. 

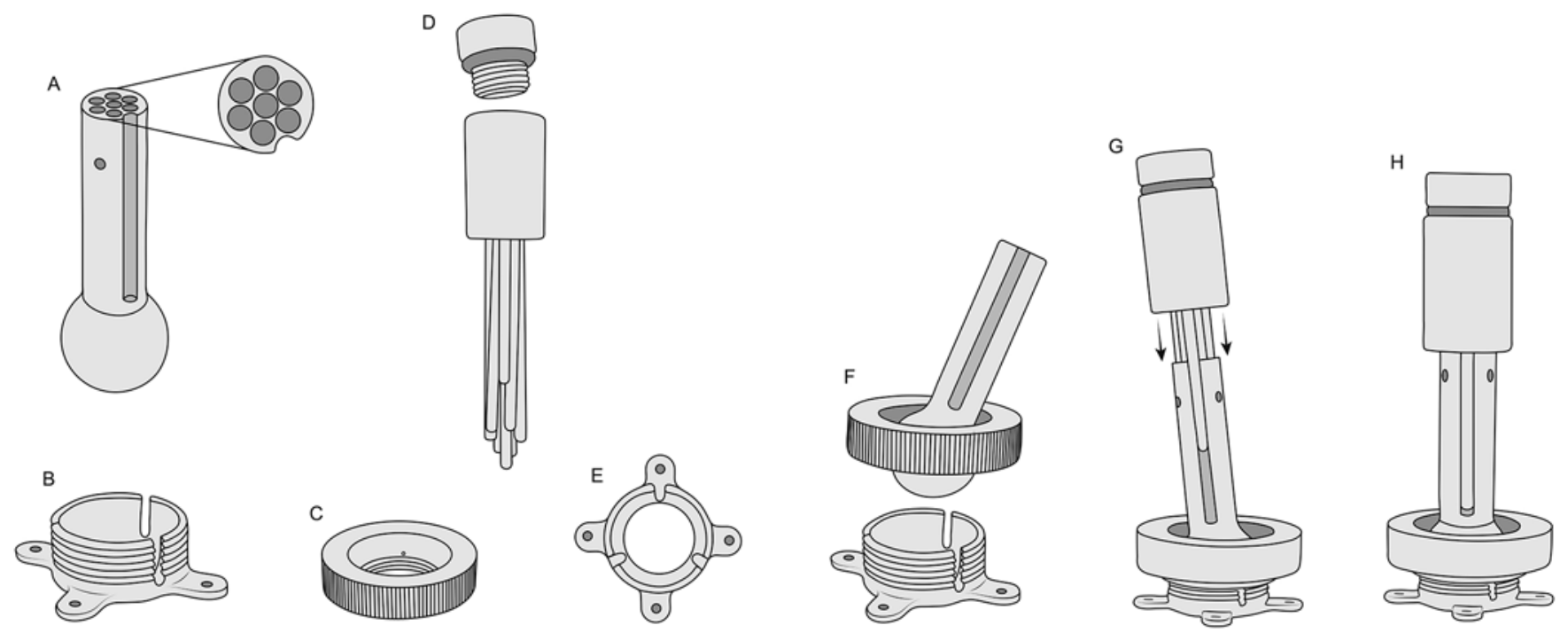

FIG. 1. Illustration of the PEEK BJGA. The BJGA stem has a 7-hole array with 2-mm-diameter array holes (A). The stem has side holes for locking side screws. A top-down view of the BJGA 7-hole stem (inset, A). The BJGA threaded base with 4 holes for titanium anchor screws that attach directly to the skull (B). The BJGA threaded collar that screws onto the skull-mounted base (C). BJGA 7-prong MRI contrast-filled fiducials with threaded cap (D). The cap is removed, and MRI contrast is placed directly into the fiducial prongs (and the cap is replaced) for imaging. Top-down view of the BJGA threaded base featured in panel B (E). The BJGA stem is placed into the threaded base and secured with the collar in position $(F)$. MRI contrast-filled fiducials are placed into the BJGA stem for MRI and intraoperative planning for cannula placement ( $\mathbf{G}$ and $\mathbf{H})$. Copyright The Ohio State University. Published with permission.

feasibility, safety, and accuracy of the BJGA, we investigated its performance during CED of adeno-associated virus (AAV) containing the $A A D C$ gene (AAV2-AADC) in AADC-deficient patients.

\section{Methods \\ Patients}

Patients with AADC deficiency undergoing MRI-guided BJGA placement of bilateral frontal infusion cannulas and image-guided direct convective delivery of AAV2AADC (with $2 \mathrm{mM}$ of gadoteridol, ProHance, Bracco Diagnostics, Inc.) were included in this study. Consent was obtained from all patients and/or their parents, under a compassionate use exemption.

\section{BJGA Apparatus}

The BJGA (Prototek and Technodiamant) is a skullmounted device fabricated from polyetheretherketone (PEEK) and adapted from a previously described preclinical device. ${ }^{14}$ It is gas sterilized and reusable and is on the path to commercialization. The BJGA has four components, including 1) a threaded base with 4 holes for titanium anchor screws that attaches directly to the skull; 2 ) a 7-pronged contrast-filled fiducial; 3) a 7-hole ball array stem with 2-mm-diameter array holes (with locking side screws for cannulas) that accepts an infusion cannula, biopsy needle, or MRI contrast-filled fiducials; and 4) a threaded collar that screws into the skull-mounted base and secures the ball array stem in position (Fig. 1). The BJGA has a $360^{\circ}$ range of rotation and maximal angulation of $27^{\circ}$ from the vertical.

\section{Targeting Accuracy, Volume of Distribution, and Statistical Analysis}

Previously, we described a method for assessing and calculating the targeting accuracy for intraoperative frameless MRI-guided stereotactic systems. ${ }^{11,15}$ Briefly, the accuracy of infusion cannula tip placement as compared to the intended target is assessed by three independent evaluators by measuring depth and radial errors and then calculating the absolute tip error. Depth and radial errors are derived using measurements directly obtained from intraoperative MRI of the cannula tip compared to the planned target. Linear correlation of error measurements was calculated using the Pearson product-moment correlation coefficient. ${ }^{16}$

\section{Technique}

\section{Preoperative Planning}

Patients undergo preoperative 3D volumetric MRI with and without contrast (TR $2300 \mathrm{msec}$, TE $5 \mathrm{msec}$, flip angle $9^{\circ}$, FOV $250 \times 250 \times 250 \mathrm{~mm}$, slice thickness $1 \mathrm{~mm}$ ). Images are loaded into a navigation workstation (Brainlab AG). Bilateral skull entry points and infusion targets are determined through orthogonal and oblique views in all three planes. Specifically, the initial cannula tip placement site ("target") is defined along a path that provides effective perfusion of the treatment region (ventral tegmental area and immediately medial subjacent substantia nigra) by moving the cannula along the planned trajectory in either a caudal to rostral or a rostral to caudal ("infuse-as-you-go"17) manner during infusion (Fig. 2). 

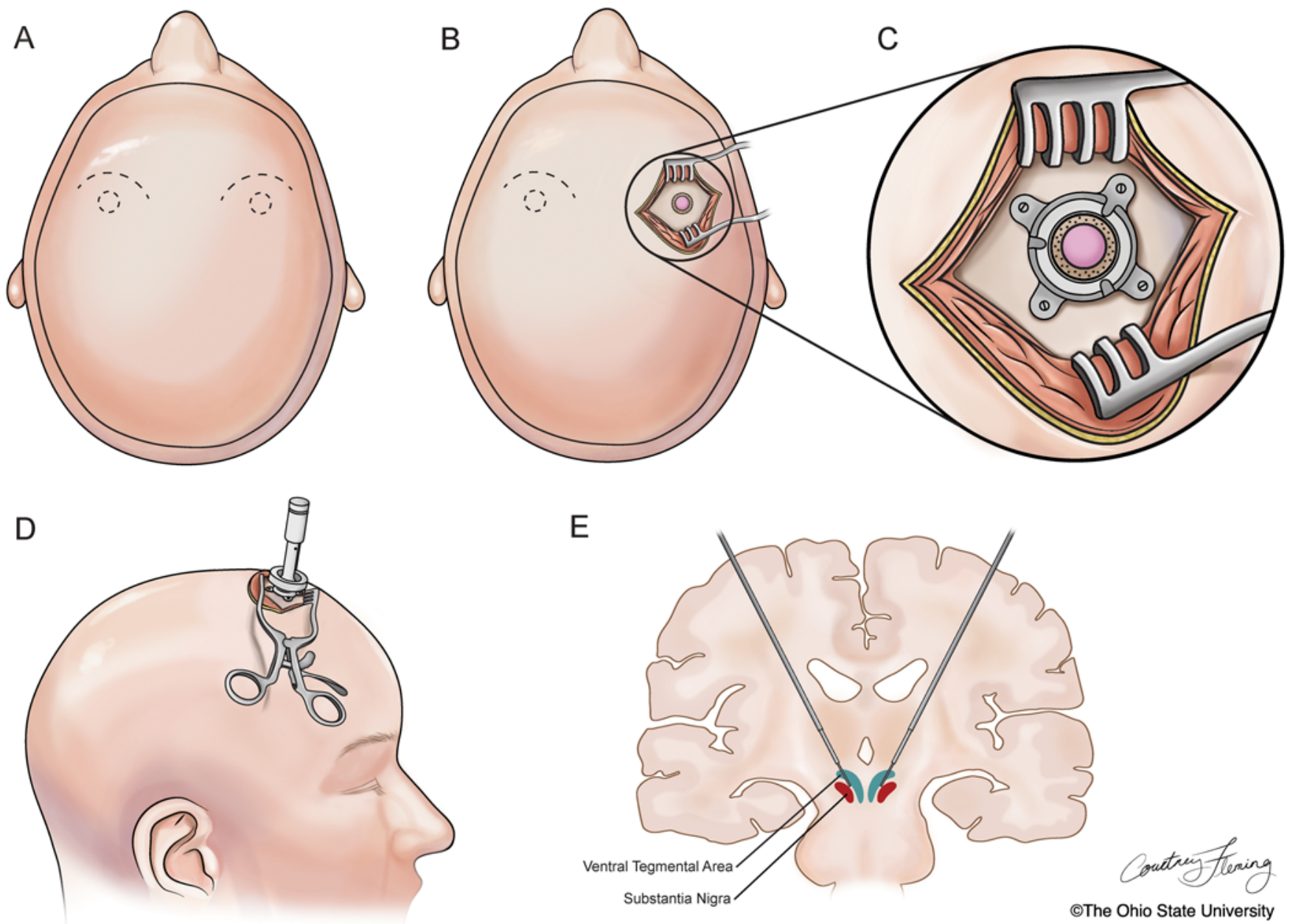

FIG. 2. Placement of BJGA for bilateral perfusion of the ventral tegmental area and substantia nigra. A: Bilateral frontal curvilinear incision sites (dashed curved lines) are placed over predetermined entry sites (burr hole, dashed circles) using frameless stereotactic navigation. B: An incision is made, and a self-retaining retractor is placed to expose the underlying skull. A burr hole is centered and created over the predefined entry point. C: Magnified view of the burr hole with the threaded base secured with four titanium screws. D: The BJGA apparatus with a 7-prong MRI contrast-filled fiducial in place is secured to the threaded base and oriented to the target using stereotactic navigation. E: After confirmation of the trajectory to the target, cannulas are placed to the target through the BJGA apparatus. Copyright The Ohio State University. Published with permission.

\section{Placement of the BJGA}

Patients are placed under general anesthesia and maintained supine. The head is pinned in a gently flexed (or neutral) position, and flex coils (Flex Small 4, Siemens) are placed on each side of the head. Incision sites are sterilely prepared and draped. Entry points are marked on the skin using navigation (Curve, Brainlab AG). The skull is exposed using a curvilinear incision that can accommodate the burr hole and the BJGA threaded base $(2-\mathrm{cm}$ width of exposed skull at the widest point). The BJGA is registered with navigation, and entry points are marked on the skull. The burr hole is centered on the entry point, and the threaded BJGA base is secured over the burr hole via four titanium screws (Fig. 2).

\section{Intraoperative Trajectory and Targeting}

The ball array stem is placed into the base and positioned precisely in the preoperative plan-defined trajecto- ry position using navigation and secured in position with the threaded collar (Fig. 2). The contrast-filled fiducials ( $2 \mathrm{mM}$ of gadoteridol, ProHance) are placed into the ball array stem, and the patient is placed in the intraoperative MRI scanner. The wounds are covered with sterile covering, and the bore of the MRI scanner is covered with a sterile drape. MRI through the entire BJGA and brain is performed on the scanner (3.0-T Magnetom Skyra, Siemens). Three-dimensional volumetric T1-weighted MRI is performed (TR $700 \mathrm{msec}$, TE $11 \mathrm{msec}$, flip angle $15^{\circ}$, FOV $210 \times 210 \times 210 \mathrm{~mm}$, slice thickness $0.8 \mathrm{~mm}$ ).

After intraoperative MRI of the BJGA and brain, the cannula trajectory is reviewed on the MRI console using an oblique multiplanar planning function. The trajectory on each side is assessed in all three axes to ensure safe placement of the cannula along the desired path. To evaluate the trajectory, lines are drawn through the center of the long axis of the fiducials (Fig. 3). When necessary, adjustments 

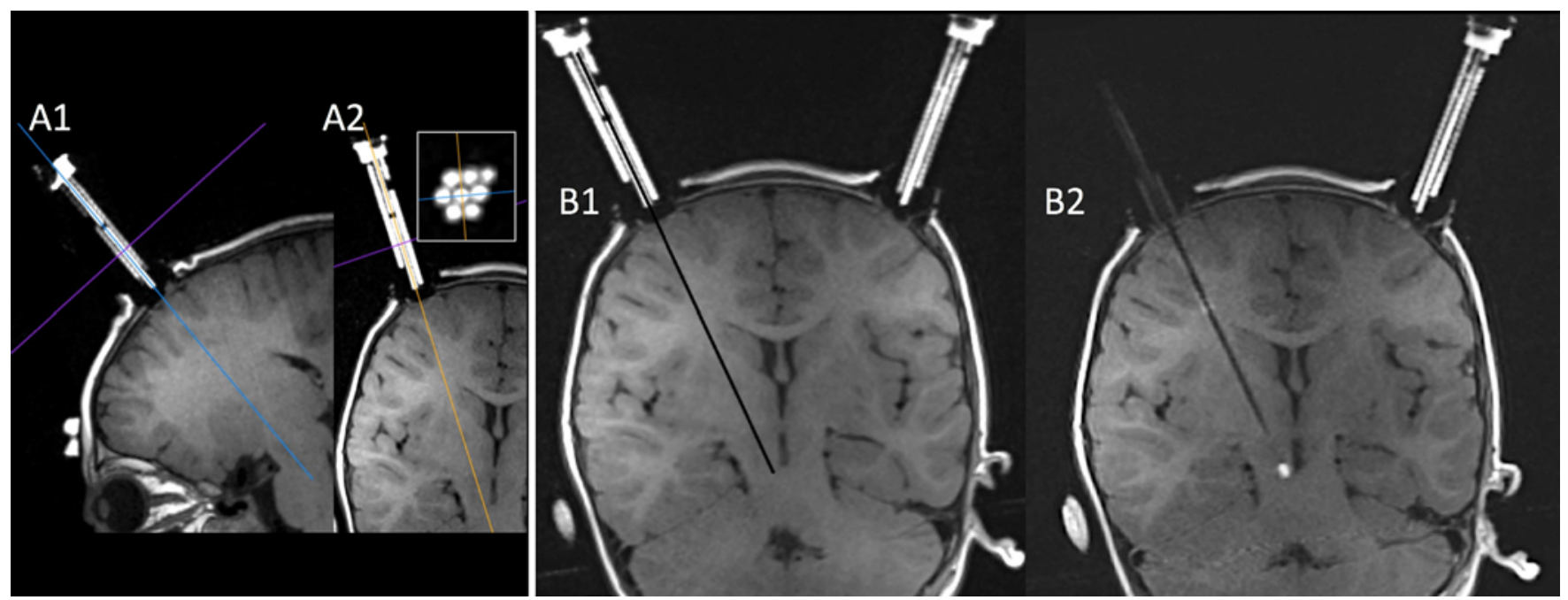

FIG. 3. Intraoperative T1-weighted MRI demonstrating planning and placement of an infusion cannula for perfusion of the ventral tegmental area and substantia nigra. Left: Intraoperative MRI of the BJGA and brain. The cannula trajectory is reviewed on the MRI console using an oblique multiplanar planning function. The trajectory on each side is assessed in all three axes and is defined by adjusting the lines that are drawn through the center of the long axis of the fiducials (A1, A2). The xyz planes are oriented such that the entire fiducial length is visible on the MRI sequences in the coronal and sagittal planes. The crosshair (inset) is placed on top of one of the fiducial signals (in this case, the middle access port shown in the axial trajectory view). Right: Planning sequence with the trajectory (136 mm from the base of the gadolinium-filled stem) drawn as shown in the left panel. Sequence reconstructed coronally (B1, B2) immediately after cannula insertion at $136 \mathrm{~mm}$. Note the contrast at the tip of the cannula at the beginning of the infusion. After infusion is completed on the one side, the procedure is repeated on the opposite side. Figure is available in color online only.

to optimize the trajectory of the array stem are confirmed by repeat MRI. Intraoperative and preoperative postcontrast T1-weighted MR images are fused to avoid vascularcannula intersection. While the planned trajectory is defined on the center hole of the ball array, any of the 7 holes can be used for optimal cannula tip placement to the target.

\section{Cannula Placement and Advancement}

Once the best trajectory and array hole for cannula placement are defined, a depth measurement along the trajectory through the array hole is made on the console from the top of the BJGA gadolinium-filled fiducial to the target. The fiducials are removed, a sharp lancet is placed through the identified hole to pierce the dura mater, and a reducer is placed in the selected access hole to accommodate the 16-gauge infusion cannula (SmartFlow catheter, MRI Interventions, Inc.). ${ }^{12} \mathrm{~A}$ stop is attached to the cannula at the measured depth distance for precise placement to the target. The cannula is advanced through the defined array hole to the target and secured with the array side screw (Fig. 2). The patient is returned to the intraoperative MRI scanner bore for cannula placement confirmation and infusion.

\section{Real-Time MRI of AAV2-AADC Delivery}

During infusion, real-time MRI is performed to monitor perfusion (Fig. 3). Once the initial target site is perfused, the side screw is loosened, and the cannula stop is adjusted to permit retraction or advancement of the cannula in 1- to 4-mm increments as determined by real-time MRI. Once the cannula is retracted or advanced, it is se- cured with the side screw and infusion continues at the new position. Cannula tip positioning along a caudal to rostral or rostral to caudal path is used to maximize target region perfusion. After complete perfusion of the treatment region, the infusion is stopped, and the cannula is left in place while a final MRI scan is obtained. Once postinfusion imaging is completed, the cannula is removed. The BJGAs are removed and the wound is closed. Patients underwent MRI 2 days after their infusion.

\section{Results}

\section{Patients}

Eleven patients ( 7 females, 4 males) underwent contemporaneous bilateral MRI-guided BJGA infusion cannula placement and CED of AAV2-AADC (22 infusions) to the brainstem (Table 1). The mean age at infusion was $10.5 \pm$ 5.2 years (range $4-19$ years).

\section{Operative Findings}

Accurate and reliable BJGA-navigated infusion cannula placement was possible in all cases. Intraoperative MRI revealed clear delineation of the gadolinium-filled fiducial in the ball stem array. This permitted precise trajectory mapping from each hole in the array and allowed for on-thefly angular adjustment of the entire array to improve the trajectory when necessary, as well as selection of the optimal array hole in translational space to pass the cannula. After the trajectory and array hole for cannula placement were confirmed, cannulas were placed at the target (single pass in all infusions). The mean time from skin incision to placement of the first infusion cannula (including bilateral 
TABLE 1. Patient and cannula placement characteristics

\begin{tabular}{|c|c|c|c|c|c|c|c|c|c|c|}
\hline $\begin{array}{l}\text { Case } \\
\text { No. }\end{array}$ & Sex & $\begin{array}{l}\text { Age } \\
\text { (yrs) }\end{array}$ & $\begin{array}{c}\text { Lt Target } \\
\text { Depth (mm) }\end{array}$ & $\begin{array}{l}\text { Rt Target } \\
\text { Depth (mm) }\end{array}$ & $\begin{array}{l}\text { Lt Radial } \\
\text { Error (mm) }\end{array}$ & $\begin{array}{l}\text { Rt Radial } \\
\text { Error }(\mathrm{mm})\end{array}$ & $\begin{array}{l}\text { Lt Depth } \\
\text { Error (mm) }\end{array}$ & $\begin{array}{l}\text { Rt Depth } \\
\text { Error (mm) }\end{array}$ & $\begin{array}{l}\text { Lt Absolute } \\
\text { Tip Error (mm) }\end{array}$ & $\begin{array}{l}\text { Rt Absolute } \\
\text { Tip Error (mm) }\end{array}$ \\
\hline 1 & $\mathrm{~F}$ & 16 & 140 & 134 & 0.4 & 1.0 & 0.7 & 0.3 & 0.8 & 1.0 \\
\hline 2 & M & 7 & 140.5 & 143.0 & 0.6 & 1.1 & 0.3 & 1.4 & 0.7 & 1.8 \\
\hline 3 & $M$ & 12 & 145.5 & 142.5 & 0.9 & 2.1 & 0.0 & 1.7 & 0.9 & 2.7 \\
\hline 4 & $F$ & 19 & 145.0 & 143.0 & 0.1 & 0.3 & 0.7 & 0.2 & 0.7 & 0.4 \\
\hline 5 & $\mathrm{~F}$ & 5 & 134.0 & 137.0 & 0.4 & 1.9 & 1.0 & 0.4 & 1.1 & 1.9 \\
\hline 6 & $\mathrm{~F}$ & 17 & 134.0 & 132.0 & 0.6 & 0.4 & 0.2 & 0.3 & 0.6 & 0.5 \\
\hline 7 & $\mathrm{~F}$ & 4 & 137.0 & 142.0 & 0.5 & 0.1 & 1.0 & 1.8 & 1.1 & 1.8 \\
\hline 8 & $M$ & 10 & 136.0 & 136.0 & 0.9 & 1.2 & 1.7 & 0.9 & 1.9 & 1.5 \\
\hline 9 & $\mathrm{~F}$ & 4 & 133.0 & 134.0 & 0.8 & 2.3 & 1.1 & 1.9 & 1.4 & 3.0 \\
\hline 10 & $\mathrm{~F}$ & 9 & 124.0 & 131.0 & 0.1 & 1.0 & 0.5 & 2.2 & 0.5 & 2.4 \\
\hline 11 & $M$ & 12 & 134.0 & 136.0 & 0.7 & 1.3 & 0.7 & 1.8 & 1.0 & 2.2 \\
\hline Mean & & 10.5 & 136.6 & 137.3 & 0.5 & 1.2 & 0.7 & 1.2 & 1.0 & 1.8 \\
\hline SD & & 5.2 & 6.1 & 4.6 & 0.3 & 0.7 & 0.5 & 0.8 & 0.4 & 0.8 \\
\hline
\end{tabular}

BJGA placement, patient transfer to intraoperative MRI scanner with MRI adjustment, and confirmation of the first cannula) was 135 minutes (range 80-200 minutes). The mean time to adjust and confirm the trajectory for the contralateral cannula was 23 minutes (range 11-55 minutes).

Following cannula placement at the target, MRI of the infusion was performed. Real-time MRI during infusion revealed a clearly defined region of AAV2-AADC distribution $^{18}$ that expanded progressively with the increasing infusion volume. Infusion imaging confirmed the adequacy of distribution and any leakage back along the cannula track (rate of infusion 1-10 $\mu \mathrm{l} / \mathrm{min}$ ). The cannula was advanced caudally or rostrally from the target in 1- to 4-mm increments along the trajectory to infuse the ipsilateral ventral tegmental area and substantia nigra (Table 1).

\section{BJGA Accuracy}

Intraoperative MRI clearly confirmed the location of the cannula tip by detection of the gadolinium coinfusate on the initial MR image after placement (all infusions; Table 1). Overall, the mean bilateral depth to the target was $137.0 \pm 5.2 \mathrm{~mm}$ (range 124.0-145.5 mm). The mean bilateral depth error was $0.9 \pm 0.7 \mathrm{~mm}$ (range $0-2.2 \mathrm{~mm}$ ), bilateral radial error was $0.9 \pm 0.6 \mathrm{~mm}$ (range $0.1-2.3 \mathrm{~mm}$ ), and bilateral absolute tip error was $1.4 \pm 0.8 \mathrm{~mm}$ (range $0.4-3.0 \mathrm{~mm}$ ). There was no correlation between target depth and absolute tip error (Pearson product-moment correlation coefficient, $r=0.01$ ).

\section{Complications}

There were no complications in the patients, including infection. MRI obtained 2 days after infusion demonstrated asymptomatic blood within the cannula tracks of 2 patients.

\section{Discussion}

\section{BJGA Design and Preclinical Assessment}

The BJGA was developed to facilitate CED cannula placement, enable on-the-fly cannula repositioning, and provide a platform for imaging infusion distribution. It was designed to overcome limitations associated with currently available MRI-compatible frameless stereotactic systems adapted (i.e., designed for deep brain stimulation lead placement) for CED. Specifically, preclinical BJGA data demonstrated advantages over these available MRI-guided frameless stereotactic systems (Table 2). ${ }^{14}$ First, the BJGA is fabricated from lightweight inexpensive

TABLE 2. Comparison of features of the BJGA and available frameless stereotactic guide systems for real-time MRI-guided CED cannula placement and infusion monitoring*

\begin{tabular}{lccc}
\hline \multicolumn{1}{c}{ Device Property } & BJGA & ClearPoint SmartFrame (ClearPoint Neuro) & Nexframe (Medtronic) \\
\hline MRI compatible & Yes & Yes & Yes \\
\hline Trajectory adjustment in MRI scanner & Yes & Yes & No \\
\hline Universal MRI software compatibility & Yes & No & No \\
\hline Multiple contemporaneous infusions & Yes & No & No \\
\hline No. of possible infusions from single frame alignment & 7 & 1 & 1 \\
\hline Dimensions (height $\times$ width in $\mathrm{cm}$ ) & $4.5 \times 2.5 \dagger$ & $15.5 \times 11.5$ & $10.8 \times 6$ \\
\hline
\end{tabular}

* Adapted from Sudhakar et al., 2020.14

† Height without guide array (height with guide array in place, $7.5 \mathrm{~cm}$ ). 
MRI-compatible material (PEEK) that provides an economical and disposable (or reusable) device for frameless stereotactic procedures. Second, because it is skull mounted, it does not require head fixation in the MRI scanner and can support unsurpassed positioning flexibility. Third, it does not require dedicated software, which reduces procedural costs, time, and complexity. Fourth, placement of this system can be performed using any navigation system or directly from MRI assessment that provides a universal application platform. Fifth, the BJGA permits on-the-fly cannula adjustment for complex perfusion architecture. ${ }^{17}$ Finally, the significantly lower profile of the BJGA permits multiple contemporaneous infusions and cannula placement across clinical MRI scanner bore sizes.

\section{Current Study \\ Patients}

All patients in this study had confirmed AADC deficiency. Given the progressive nature of this disease and its associated mortality, all patients were under the age of 19 years at treatment. All patients underwent bilateral realtime MRI confirmation of AAV2-AADC perfusion of the target region, ${ }^{18}$ which included the ventral tegmental area and substantia nigra. The depth, irregular architecture, and size of the infusion region provided an ideal opportunity to critically assess the BJGA.

\section{Feasibility}

Use of the BJGA for infusion cannula placement, stability during infusion imaging, and cannula advancement exceeds the abilities of currently available devices. The device design facilitates easy and secure mounting to the skull. The array has a $360^{\circ}$ range of rotation and available angulation of $27^{\circ}$ from vertical, providing critical adjustment flexibility. The multiple array holes allow for cannula placement optimization in translational space (Fig. 1).

The ClearPoint system can be scalp mounted with twist drill penetration of the skull for single cannula placement. While the BJGA requires a larger incision to accommodate a burr hole, the BJGA design and the use of a burr hole for intracranial cannula access provide significantly more degrees of freedom for the placement of several contemporaneous or serial cannulas to various targets without device reestablishment. A scalp-mounted BJGA is being developed for infusion cannula placement via twist drill.

The BJGA is universally compatible with all navigation systems for preoperative entry point/trajectory planning. The BJGA does not require dedicated software for intraoperative trajectory planning or adjustment. Intraoperative planning and adjustment occur on the MRI console and in the MRI scanner. These features underscore the device's ease of use and allow the BJGA to be deployed for infusions at any center with MRI capabilities. Finally, validation of the cannula trajectory after cannula introduction into the brain can be confirmed in real time.

While we did not perform a direct comparison of efficiency for the BJGA and ClearPoint systems in these patients, we have used both systems extensively for convective delivery to the brain in clinical trials. Use of the BJGA in similar infusion cases reduces total operative time by $25 \%-50 \%$. This time reduction is principally attributable to an ability to rapidly adjust the trajectory across a wide range (degrees of freedom) and to optimize the trajectory through the multiple cannula ports (mean time for BJGA trajectory adjustment, 23 minutes).

\section{Accuracy}

The BJGA was accurate and reliable for placing infusion cannulas to the target in all cases after one pass. Preclinical BJGA data have demonstrated that this device is extremely accurate for the placement of an infusion cannula in nonhuman primates (mean cannula tip error, 1.2 $\mathrm{mm}) \cdot{ }^{14}$ Consistent with these data, we found a mean absolute tip error of $1.4 \mathrm{~mm}$ over all infusions. These findings compare favorably with those with available frameless stereotactic systems that have been adapted for CED cannula placement and CED (Table 2). ${ }^{11,15,19}$ The lack of systematic errors in cannula placement underscores the fidelity and accuracy of the BJGA.

\section{Effectiveness}

The BJGA was effective in cannula placement and advancement along the planned trajectory for perfusion of the irregularly shaped treatment region (Table 1). The ability to perform on-the-fly changes in the cannula trajectory and translational position based on real-time MRI was critical for successful perfusion. Not only did the BJGA provide accurate cannula placement, it permitted effective caudal to rostral or rostral to caudal (infuse-as-you-go ${ }^{17}$ ) delivery along the planned trajectory that maximized perfusion of the treatment region.

\section{Clinical Features}

While multiple contemporaneous infusions were not performed in these patients, the low profile of the BJGA (Table 2) allowed the simultaneous placement of bifrontal devices. Future trials requiring two or more sites of infusion will make it essential to place multiple devices on the skull for simultaneous infusions and/or multiple infusions. Multiple access holes in the BJGA permit multiple simultaneous infusions in targets at variable depths (e.g., thalamus or brain tumors) through the same array. These features are not possible with other available systems (Table 2).

\section{Safety Features}

The use of the BJGA was safe in these patients. There were no device-associated complications. Because the BJGA is made from PEEK, it is safe for use in 3.0-T MRI scanners (or higher field).

\section{Conclusions}

Intraoperative MRI-guided use of the BJGA for infusion cannula placement, perfusion monitoring, and cannula adjustment during convective delivery is feasible, effective, safe, and accurate. The BJGA provides advantages over other available frameless stereotactic systems for MRI-guided CED.

\section{Acknowledgments}

We acknowledge the clinical support of Rafal Wojdacz, MD, and Renata Serwinska (Brodno Hospital) and technical support 
from Dr. Malgorzata Kohutnicka and Technodiamant, LLC, Warsaw, Poland.

\section{References}

1. Bobo RH, Laske DW, Akbasak A, et al. Convection-enhanced delivery of macromolecules in the brain. Proc Natl Acad Sci U S A. 1994;91(6):2076-2080.

2. Lonser RR, Sarntinoranont M, Morrison PF, Oldfield EH. Convection-enhanced delivery to the central nervous system. J Neurosurg. 2015;122(3):697-706.

3. Valles F, Fiandaca MS, Eberling JL, et al. Qualitative imaging of adeno-associated virus serotype 2-human aromatic L-amino acid decarboxylase gene therapy in a phase I study for the treatment of Parkinson disease. Neurosurgery. 2010; 67(5):1377-1385.

4. Heiss JD, Lungu C, Hammoud DA, et al. Trial of magnetic resonance-guided putaminal gene therapy for advanced Parkinson's disease. Mov Disord. 2019;34(7):1073-1078.

5. Warren Olanow C, Bartus RT, Baumann TL, et al. Gene delivery of neurturin to putamen and substantia nigra in Parkinson disease: a double-blind, randomized, controlled trial. Ann Neurol. 2015;78(2):248-257.

6. Nutt JG, Curtze C, Hiller A, et al. Aromatic L-amino acid decarboxylase gene therapy enhances levodopa response in Parkinson's disease. Mov Disord. 2020;35(5):851-858.

7. Souweidane MM, Kramer K, Pandit-Taskar N, et al. Convection-enhanced delivery for diffuse intrinsic pontine glioma: a single-centre, dose-escalation, phase 1 trial. Lancet Oncol. 2018;19(8):1040-1050.

8. Vogelbaum MA, Aghi MK. Convection-enhanced delivery for the treatment of glioblastoma. Neuro Oncol. 2015;17(suppl 2):ii3-ii8.

9. Vogelbaum MA, Brewer C, Barnett GH, et al. First-in-human evaluation of the Cleveland Multiport Catheter for convection-enhanced delivery of topotecan in recurrent high-grade glioma: results of pilot trial 1. J Neurosurg. 2019;130(2): 476-485.

10. Niethammer M, Tang CC, Vo A, et al. Gene therapy reduces Parkinson's disease symptoms by reorganizing functional brain connectivity. Sci Transl Med. 2018;10(469):eaau0713.

11. Chittiboina P, Heiss JD, Lonser RR. Accuracy of direct magnetic resonance imaging-guided placement of drug infusion cannulae. J Neurosurg. 2015;122(5):1173-1179.

12. Richardson RM, Kells AP, Martin AJ, et al. Novel platform for MRI-guided convection-enhanced delivery of therapeutics: preclinical validation in nonhuman primate brain. Stereotact Funct Neurosurg. 2011;89(3):141-151.

13. Starr PA, Martin AJ, Larson PS. Implantation of deep brain stimulator electrodes using interventional MRI. Neurosurg Clin N Am. 2009;20(2):193-203.
14. Sudhakar V, Mahmoodi A, Bringas JR, et al. Development of a novel frameless skull-mounted ball-joint guide array for use in image-guided neurosurgery. J Neurosurg. 2020;132(2): 595-604.

15. Mohyeldin A, Lonser RR, Elder JB. Real-time magnetic resonance imaging-guided frameless stereotactic brain biopsy: technical note. J Neurosurg. 2016;124(4):1039-1046.

16. Shieh G. Estimation of the simple correlation coefficient. Behav Res Methods. 2010;42(4):906-917.

17. Sudhakar V, Naidoo J, Samaranch L, et al. Infuse-as-you-go convective delivery to enhance coverage of elongated brain targets: technical note. J Neurosurg. 2020;133(2):530-537.

18. Richardson RM, Kells AP, Rosenbluth KH, et al. Interventional MRI-guided putaminal delivery of AAV2-GDNF for a planned clinical trial in Parkinson's disease. Mol Ther. 2011; 19(6):1048-1057.

19. Larson PS, Starr PA, Bates G, et al. An optimized system for interventional magnetic resonance imaging-guided stereotactic surgery: preliminary evaluation of targeting accuracy. Neurosurgery. 2012;70(1 Suppl Operative):95-103.

\section{Disclosures}

Dr. Bankiewicz holds the patent for the ball-joint guide array device and is a founder and chief executive officer (stockholder) for Brain Neurotherapy Bio, Inc., which holds the exclusive license for this technology. Dr. Lonser is a scientific founder (stockholder) of Brain Neurotherapy Bio, Inc.

\section{Author Contributions}

Conception and design: Bankiewicz, Lonser, Zabek. Acquisition of data: all authors. Analysis and interpretation of data: Bankiewicz, Kreatsoulas, Onikijuk, Mozgiel, Munjal, Lonser, Zabek. Drafting the article: Bankiewicz, Elder, Lonser. Critically revising the article: Bankiewicz, Kreatsoulas, Elder, Lonser, Zabek. Reviewed submitted version of manuscript: Bankiewicz, Pasterski, Kreatsoulas, Onikijuk, Mozgiel, Elder, Lonser, Zabek. Approved the final version of the manuscript on behalf of all authors: Bankiewicz. Statistical analysis: Bankiewicz, Munjal. Administrative/technical/material support: all authors. Study supervision: Bankiewicz, Lonser, Zabek.

\section{Correspondence}

Krystof S. Bankiewicz: The Ohio State University Wexner Medical Center, Columbus, OH. krzysztof.bankiewicz@osumc. edu. 